\title{
Combined femoral and sciatic nerve block with Levobupivacaine in Brugada syndrome patient: A case report
}

\author{
Diego Tavoletti ${ }^{1}$, Elisabetta Rosanò ${ }^{1}$, Elisabetta Cerutti ${ }^{2}$, Luca Pecora ${ }^{2}$
}

\section{Abstract}

Introduction: Brugada syndrome is a rare arrhythmic disorder associated to ventricular fibrillation and sudden cardiac death. General recommendations in Brugada syndrome patient are focused on avoiding increases in vagal tone and precipitating agents such as electrolyte disorders, hypo/hyperthermia and drugs. Anesthetic management in Brugada syndrome patient has been described in many case reports, however it is still unknown which anesthesia is the safest. Local anesthetic may have a theoretical arrhythmogenic risk, but currently there is no clear evidence and their risk is still debated. Peripheral nerve block avoids autonomic nervous system changes that occur with other anesthetic techniques and it is associated to a lower level in the plasma concentration of the local anesthetic compared with neuraxial anesthesia.

Case Report: We report a case of a 38-year-old man patient with Brugada syndrome who underwent urgent orthopedic surgery which was managed with a combined femoral and sciatic nerve blocks using levobupivacaine without any complications.

Conclusions: Peripheral nerve block with levobupivacaine provided good analgesia, hemodynamic and cardiac stability in Brugada syndrome patient.

Keywords: Brugada syndrome, Anesthesia, Levobupivacaine, Peripheral nerve block, Regional anesthesia

\section{Introduction}

Brugada syndrome $(\mathrm{BrS})$ is a cardiac rhythm disorder due to mutations in genes that code for the subunits of sodium, potassium and calcium channels or for proteins that regulate these channels in the heart. It predominantly a f f e c t s young people. A n electrocardiographic (ECG) pattern characterized by a prominent coved STsegment elevation $\geq 2 \mathrm{~mm}$ followed by a negative $T$ wave in the right precordial leads (Brugada type 1 pattern) is diagnostic of $\mathrm{BrS}$. A right bundle branch block may be present, but is not required for the diagnosis. Similarly, types 2 and 3 ECG patterns are suggestive but not confirmatory [1]. Type of anesthesia, drugs and some clinical conditions can trigger the Brugada ECG pattern or favor the onset of ECG abnormalities, lethal arrhythmias, or sudden cardiac death (SCD) [2].
We report the anesthetic management of a $\mathrm{BrS}$ patient who required urgent orthopedic surgery.

Few cases of anesthetic management with peripheral nerve block (PNB) are described in $\mathrm{BrS}$ patients. However, to our knowledge, this is the first PNB performed in the lower limb with levobupivacaine. Written informed consent was obtained from patient for this report.

\section{Case Report}

A 38-year-old man (height: $178 \mathrm{~cm}$, weight: $76 \mathrm{~kg}$ ) was admitted to hospital after a motorcycle accident. His medical history was unremarkable and he did not take medications.

Physical examination demonstrated that he was awake, alert and oriented and that he had suffered no neurological injury. He exhibited multiple diffuse abrasions and an open fracture dislocation of the right ankle (Fig. 1). His vital parameters remained stable throughout the examination. ECG disclosed a Brugada type 1 pattern that was unknown to him (Fig. 2).

His history was negative for syncope and there was no case of SCD in the family. His blood chemistry test results were in the normal range.

Radiological examination revealed a fracture of the 6th cervical vertebra and a fracture dislocation of the right ankle. Spinal surgery was excluded by the neurosurgical evaluation, and the cervical spine was stabilized with a philadelphia collar. Orthopedic examination demonstrated the urgent need for an external limb fixator.

A transthoracic echocardiogram showed a normal heart with an ejection fraction of $65 \%$.

Anesthesiologist decided to manage the

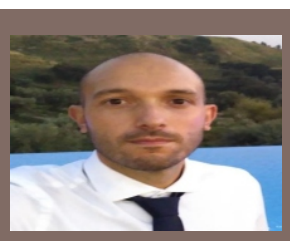

Dr. Diego Tavoletti

(C) 2020 by Journal of Anaesthesia and Critical Care Case Reports|Available on www.jaccr.com | DOI: 10.13107/jaccr.2020.v06i01.149

This is an Open Access article distributed under the terms of the Creative Commons Attribution Non-Commercial License (http://creativecommons.org/licenses/by-nc/3.0) which permits unrestricted non-commercial use, distribution, and reproduction in any medium, provided the original work is properly cited. 


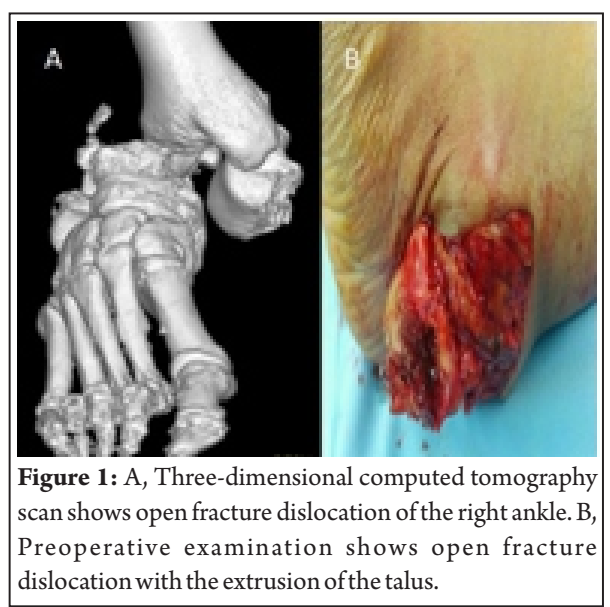

patient with a PNB, because it would allow him to lie in supine decubitus, thus avoiding a possible spine mobilization during orotracheal intubation or spinal anesthesia execution.

In the preoperative room, ECG and pulse oximetry were established and an arterial radial cannula was inserted under local anesthesia to monitor blood pressure. Defibrillator pads were applied to the chest. Intravenous midazolam $(0.05 \mathrm{mg} / \mathrm{kg})$ was administered for patient comfort.

Femoral nerve block and sciatic nerve block (SNB) were performed in aseptic conditions under ultrasound system (Sonosite SII, Bothell, WA, USA) with a needle in plane approach and a nerve stimulator (Stimuplex HNS11, B. Braun, Melsungen, Germany), to ensure correct nerve identification and minimize the dose of local anesthetic.

The femoral nerve was identified through an ultrasound probe $(6-13 \mathrm{MHz}$, Sonosite SII, Bothell, WA, USA) lateral to the femoral artery and just below the inguinal ligament. A 22 gauge, $50 \mathrm{~mm}$ insulated needle (Stimuplex A, B. Braun, Melsungen, Germany) connected to a nerve stimulator was inserted and advanced toward the nerve. When a current of $0.3 \mathrm{~mA}$ elicited a twitch of the quadriceps muscle and a 'dancing patella' sign, $10 \mathrm{ml} 0.5 \%$ levobupivacaine was injected after repeated negative aspiration for blood.

The SNB was performed by the anterior approach with the patient supine, the hip abducted and externally rotated, and the knee flexed. The ultrasound probe $(2-5 \mathrm{MHz}$, Sonosite SII, Bothell, WA, USA) was positioned perpendicular to the lesser trochanter and the sciatic nerve was identified as a hyperechoic structure posterior and medial to it. A 22 gauge, 120 mm insulated needle (Stimuplex D, B. Braun,
Melsungen, Germany) connected to a nerve stimulator was inserted and advanced toward the nerve. When a current of $0.3 \mathrm{~mA}$ evoked a motor response (foot plantar flexion) in the gastrocnemius muscle, $12 \mathrm{ml} 0.5 \%$ levobupivacaine was injected after repeated negative aspiration for blood.

Body temperature and plasma electrolytes were monitored throughout the procedure. Normothermia was maintained with a thermal blanket.

Surgical time was 140 minutes and the external fixator was successfully applied.

Neither arrhythmia or hemodynamic changes were observed during intraoperative period.

At the end of surgery, the patient's visual analogue scale (VAS) was $0 / 10$. After one hour he was admitted in intensive care unit, where continuous ECG monitoring was performed for $36 \mathrm{~h}$.

After 12 hours the VAS score was 3/10 and a paracetamol $1 \mathrm{~g}$ was administered. No other analgesic drugs were required.

Postoperative period was uneventful, vital signs were stable. He was discharged after 9 day to the surgery.

\section{Discussion}

General recommendations in $\mathrm{BrS}$ patient are focused on avoiding increases in vagal tone and precipitating agents such as electrolyte disorders, hypo/hyperthermia and drugs. Currently, the basis for considering these drugs as prohibited in $\mathrm{BrS}$ patients is weak and there isn't strong evidence.

Besides, anesthetic management in $\mathrm{BrS}$ patient has been described in many case reports, however which is the safest anesthesia is still unknown [1].

Traditionally, regional anesthesia is discouraged as local anesthetic (LA) causes a sodium channels blockade, increasing the risk of arrhythmias in $\mathrm{BrS}$ patients.

Neuraxial anesthesia (NA) involves a degree of risk, because it can unmask the arrhythmia, as demonstrated in two patients whose BrS was revealed by the epidural block with bupivacaine and spinal anesthesia with prilocaine $[3,4]$. Moreover, ventricular fibrillation after epidural anesthesia and ventricular tachycardia after paravertebral block with ropivacaine, were reported in two $\mathrm{BrS}$ patients $[5,6]$. However, uneventful epidural and spinal anesthesia are described $[1,7,8]$.

It is impossible to affirm that the complications were direct action of LA in ion

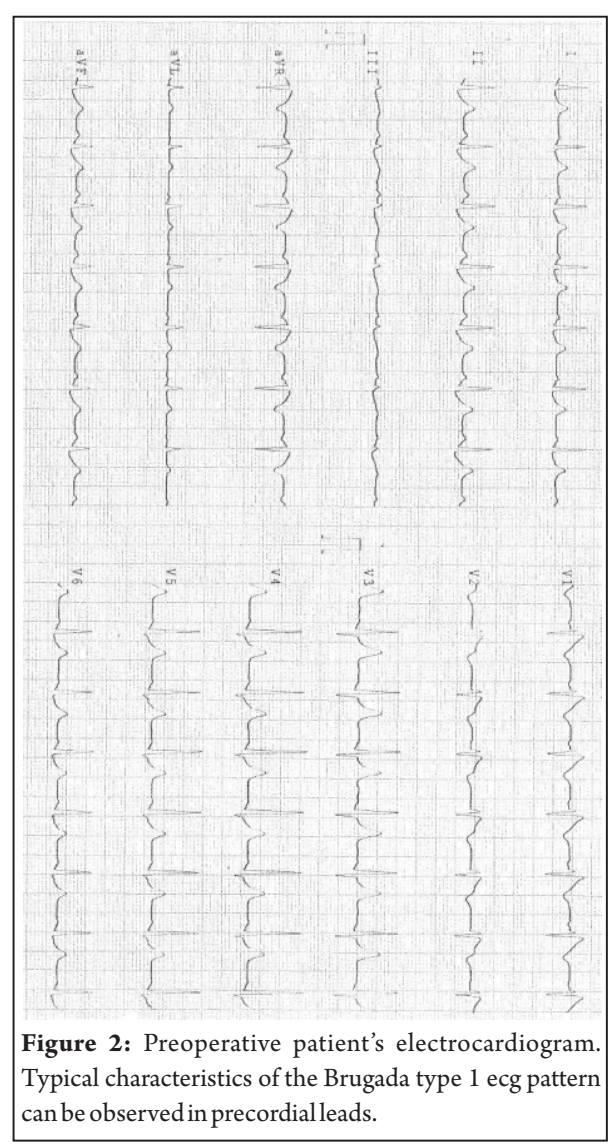

channels, because complications could be due to the autonomic nervous system changes after epidural and spinal anesthesia.

Few cases of anesthetic management with PNB are described in BrS patients. Three brachial plexus blocks and an ilioinguinal nerve block have successfully been performed [9-12].

We preferred to manage the patient with PNB, because it allowed us to keep the patient in a supine position (due to cervical vertebra fracture) and avoid autonomic nervous system effects that occur with other anesthetic techniques.

The autonomic nervous system affects ion channel activity. A careful selection of anesthesia and analgesia medications is required to minimize autonomic nervous system stimulation, avoiding the ECG changes typical of $\mathrm{BrS}$ [13]. General and NA are known to induce rapid autonomic nervous system changes, adding to the risk of tachyarrhythmia in BrS patients [3,8]. PNB does not cause rapid autonomic nervous system effects. Furthermore, it affords better postoperative analgesia, limiting the autonomic nervous system stimulation due to pain [2].

The rapidity of absorption, type and dose of LA could determine the safety profile of 
regional anesthesia. NA is associated to a faster increase in the plasma concentration of the LA compared with PNB. The cases where local anesthetics have been implicated in the development of Brugada ECG abnormalities and cardiovascular instability were probably related to rapid absorption of the agent into the systemic circulation. Notably, negative aspiration during PNB avoids accidental intravascular injection of the LA, while ultrasound guidance allows reducing the LA dose.

LA may have a theoretical arrhythmogenic risk in $\mathrm{BrS}$ patient, but currently there is no clear evidence. A list of drugs that are preferably to be avoided has been published however their risk is still debated.

Conflicting reports on the safety of the ropivacaine or bupivacaine administration have been previously described.

The use of lidocaine for local anesthesia has been shown to be not dangerous in some cases [1].
We had chosen levobupivacaine (a long acting LA) for postoperative analgesia, to prevent sudden changes in autonomic tone due to pain.

Levobupivacaine, the pure $S(-)$-enantiomer of bupivacaine, has been shown to be a safer alternative to the racemic form. In animal studies, it demonstrated less inhibitory effect on the cardiac sodium channels and less i $\mathrm{m} \mathrm{p}$ a i r m e $\mathrm{t}$ of $\mathrm{th}$ e h e a $\mathrm{t}$ conduction/contractile functions. Minor effects have been observed on cloned human heart sodium and potassium channels. In human studies, levobupivacaina showed a lower volume of distribution, an increased protein-binding affinity and a higher clearance of the unbound fraction than dextrobupivacaine. This explains the shorter elimination half-life of $S(-)$-bupivacaine [14].

For sedation, intravenous drugs such as midazolam and propofol have been used without problems in several cases, although prolonged propofol infusion has been implicated in revealing $\mathrm{BrS}[7]$.

To our knowledge, this is the first anesthetic management with PNB in the lower limbs in $\mathrm{BrS}$ patient. Furthermore, no cases of levobupivacaine administration are reported in $\mathrm{BrS}$ patients.

In conclusion, LA in nerve blocks with large systemic absorption, such as NA, should be used carefully. Agents that could trigger arrhythmias, such as autonomic nervous system changes, should be avoided. PNB with levobupivacaine seems to provide a safe approach in these patients. In addition, the combination of nerve stimulation and ultrasound allows reducing the LA dose. Additional clinical research are required to demonstrate the safety of PNB.

\section{References}

1.Duque M, Santos L, Ribeiro S, Catré D. Anesthesia and Brugada syndrome: A 12-year case series. JClin Anesth. 2017;36:168-73.

2. Carey SM, Hocking G. Brugada syndrome: a review of the implications for the anaesthetist. Anaesth Intensive Care. 2011;39:571-77.

3. Phillips N, Priestley M, Denniss AR, Uther JB. Brugada-type electrocardiographic pattern induced by epidural bupivacaine. Anesth Analg. 2003;97:264-67.

4. Oliván B, Arbeláez A, de Miguel M, Pelavski A. Diagnosis of Brugada's syndrome after subarachnoid injection of prilocaine. Rev Esp Anestesiol Reanim. 2016;9356:278-79.

5. Kaneda Y, Fujita N, Ueda K, Saeki K, Sakano H, Sudo M, et al. Surgically treated primary lung cancer associated with Brugada syndrome: report of a case. Surg Today. 2001;31:817-19.

6. Fujiwara Y, Shibata Y, Kurokawa S, Satou Y, Komatsu T. Ventricular tachycardia in a patient with Brugada syndrome during general anesthesia combined with thoracic paravertebral block. Anesth Analg. 2006;102:1590-1.

7. Kloesel B, Ackerman MJ, Sprung J, Narr BJ, Weingarten TN. Anesthetic management of patients with Brugada syndrome: a case series and literature review. Can JAnaesth. 2011;58:824-36.

8. Kim JS, Park SY, Min SK, Kim JH, Lee SY, Moon BK. Anaesthesia in patients with Brugada syndrome. Acta Anaesthesiol Scand. 2004;48:1058-61.

9. Bang YS, Lee SY, Ko D, , Park J, Ahn S, Park C. Brugada syndrome patient undertaken brachial plexusblock. EwhaMedJ.2017;40:164-7.

10. Vasques F, Di Gregorio G, and Behr AU. Is prevention better than cure? local anesthetics in Brugadasyndrome. Regional Anesth Pain Med. 2015;40:395-6.

11. Chen Q, Venkatesan K, Cheong G. General and regional anaesthesia in Brugada Syndrome - a case report. Trends in Anaesthesia and Critical Care 2017.

12. Ferreira MN, Fontes S, Machado H. Interscalene plexus block and general anesthesia in Brugada syndrome. SaudiJAnaesth. 2019;13:371-3.

13. Dulu A, Pastores SM, McAleer E, Voigt L, Halpern NA. Brugada electrocardiographic pattern in a postoperative patient. Crit Care Med 2005;33:1634-7.

14. Burlacu CL, Buggy DJ. Update on local anesthetics: Focus on levobupivacaine. Ther Clin Risk Manag. 2008;4:381-92.
Conflict of Interest: Nil

Source of Support: None

\section{How to Cite this Article}

Tavoletti D, Rosanò E, Cerutti E, Pecora L | Combined femoral and sciatic nerve block with Levobupivacaine in Brugada syndrome patient: A case report | Journal of Anaesthesia and Critical Care Case Reports |Jan-April 2020; 6(1): 19-21. 